\title{
Membership and structural parameters of the globular cluster M 5 from Schmidt plates
}

\author{
N. Kharchenko ${ }^{1}$, R.-D. Scholz ${ }^{2}$, and I. Lehmann ${ }^{3,4}$ \\ 1 Main Astronomical Observatory, Golosiiv, 252127 Kiev, Ukraine \\ 2 WIP Astronomie / Universität Potsdam, Sternwarte Babelsberg, An der Sternwarte 16, D-14482 Potsdam, Germany \\ 3 Humboldt-Universität zu Berlin, Germany \\ 4 Astrophysikalisches Institut Potsdam, An der Sternwarte 16, D-14482 Potsdam, Germany
}

Received March 26; accepted June 6, 1996

\begin{abstract}
Using absolute proper motions derived from APM measurements of Tautenburg, Palomar and UK Schmidt plates (cf. Scholz et al. 1996) we determined membership probabilities of the globular cluster M 5 in a four-dimensional space of proper motions and coordinates. The solution for 19 unknown parameters was obtained by the maximum likelyhood method. Different mean absolute proper motions and dispersions were found for three distribution functions of the cluster stars, near field stars and distant field stars. In addition to the four-dimensional distribution functions, we also investigated two-dimensional distribution functions including either only the proper motions or only the coordinates. We compared the results with the colour-magnitude diagram obtained from a $B, V$ photographic photometry using two of the Tautenburg plates and with the membership determination of Rees (1993). In comparison to the last work based on observations with larger epoch differences and better astrometric scale, our results are less accurate but extend the membership investigation of M 5 to fainter stars $(B=19)$ and to the outer regions of the cluster. The full APM scans of the different Schmidt plates were also used for star counts applying a crowding correction in the central cluster region based on the area of crowded images and on the stellar luminosity function in the outer cluster region. The tidal radii from fitting King profiles to the counts of all objects on the plates and of the cluster members are discussed. From the deep counts of the full scans we found an indication of a tidal tail around M 5 (cf. Grillmair et al. 1995). However, this effect was not confirmed by the member counts.
\end{abstract}

Key words: astrometry - methods: statistical globular clusters: individual: M 5 - stars: kinematics

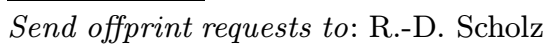

\section{Introduction}

The globular cluster M 5 is one of the clusters investigated within our programme of determining mean absolute proper motions and Galactic orbits of globular clusters (Scholz et al. 1993, 1994; Scholz et al. 1996). This programme is based on Schmidt plates with epoch differences between 20 and 40 years. The main aim of the programme is to measure accurate mean absolute proper motions of globular clusters directly with respect to an extragalactic reference frame represented by large numbers of background galaxies. For this purpose the Schmidt plates are measured by the Automated Plate Measuring System (APM) (Kibblewhite et al. 1984) in order to fully extract all the information available on the plates.

Accurate membership determinations of many globular clusters based on proper motions have been obtained by Cudworth and coworkers (e.g. Cudworth 1979). In their investigations they used refractor plates with a good astrometric scale $(\sim 10 \operatorname{arcsec} / \mathrm{mm})$ taken over a baseline of more than 70 years so that they obtained very high accuracies in the relative proper motions (of the order of several $100 \mu \mathrm{as} / \mathrm{yr}$ ). Due to the smaller epoch difference and the much larger scale factor of the Schmidt plates $(\sim 50 \operatorname{arcsec} / \mathrm{mm})$ we can only achieve proper motion accuracies for single stars on the level of several mas/yr. On the other hand, the Schmidt plates go much deeper and cover the whole cluster region in comparison to the known membership studies, which are usually restricted to the bright cluster stars $(V<16)$ and the central cluster region $(r<10 \operatorname{arcsec})$. For the globular cluster M 3 we have already demonstrated the possibility of extending membership investigations to the faint stars and to the outer cluster regions by using Tautenburg Schmidt plates (Scholz \& Kharchenko 1994).

The globular cluster M 5 presents a rich outer halo cluster in the special state of a close encounter near the Galactic center. This conclusion may be drawn from the 
high space velocity resulting from the combination of the present heliocentric distance, the radial velocity and absolute proper motion results. A large absolute proper motion of M 5 had already been mentioned by Cudworth (1979) converting the mean relative proper motion of the cluster to an absolute one via the secular parallax of faint field stars in the cluster vicinity. Cudworth \& Hanson (1993) used the more accurate relative proper motion results of Rees (1993) confirming an unusually high space velocity of the cluster. Although the new absolute proper motion of M 5 measured directly with respect to more than 1000 reference galaxies (Scholz et al. 1996) is somewhat smaller than given by Cudworth \& Hanson (1993) the space velocity remains large $(420 \mathrm{~km} / \mathrm{s})$. The resulting orbit of M 5 on the basis of the Galactic model of Allen \& Santillan (1991) leads through the outskirts of the Galaxy with an apogalacticon of about $60 \mathrm{kpc}$. (With the former velocity values from Cudworth \& Hanson (1993) the apogalactic distance of M 5 would even rise to $140 \mathrm{kpc}$.) The large absolute proper motion of M 5 allows to distinguish the proper motion distribution of the cluster from that of the field stars even if we plot the results from the Schmidt data in a vector-point proper motion diagram (see Fig. 4a in Scholz et al. 1996).

Here we use the proper motion results of Scholz et al. (1996) in membership determinations in a fourdimensional space of proper motions and coordinates with the maximum likelyhood method. The results are compared with the membership determination from twodimensional distribution functions including only the proper motions or only the coordinates and with the colour-magnitude diagram (CMD) obtained from a $B, V$ photographic photometry using two of the Tautenburg plates. The membership probabilities of the brighter cluster stars in the central cluster region obtained by Rees (1993) on the basis of proper motions with an order of magnitude better accuracies than ours provide a good external check of the reliability of our membership determinations.

Palomar Schmidt plates have already been used for star counts in many globular clusters, including M 5, by King et al. (1968). First attempt to use automated measuring machines for that task were made by Herzog \& Illingworth (1977). Irwin \& Trimble (1984) measured the southern globular cluster M 55 on plates of the $150^{\prime \prime}$ telescope of the Anglo-Australian Observatory with the APM measuring machine. Recently, Grillmair et al. (1995) examined the outer structure of 12 Galactic globular clusters using star counts on UK Schmidt plates scanned with the APM.

Tautenburg Schmidt plates measured with the APM were used in an investigation of the density profiles and luminosity functions of the globular clusters M 3 and M 92 (Scholz \& Schmidt 1992). APM scans of different Schmidt plates with seven other globulars included in the absolute proper motion programme were also used for star counts applying a crowding correction in the central cluster region based on the area of crowded images and the luminosity function in the outer cluster region (Lehmann 1996). Here we discuss the tidal radii of M 5 obtained from fitting King profiles to the counts of all objects on single plates and to the counts of the cluster members. Member counts had already been used by Tucholke (1992) in order to improve the determination of tidal radii of the globular clusters 47 Tuc and NGC 362.

Table 1. Plate material

\begin{tabular}{ccccc}
\hline Telescope & $\begin{array}{c}\text { Pass- } \\
\text { band }\end{array}$ & $\begin{array}{c}\text { exp. } \\
{[\mathrm{min}]}\end{array}$ & $\begin{array}{c}\text { Scale } \\
{[\operatorname{arcsec} / \mathrm{mm}]}\end{array}$ & Epoch \\
\hline POSSI/O1402 & $U+B$ & 12 & 67.2 & 1955.30 \\
POSSI/E1402 & $R$ & 45 & 67.2 & 1955.30 \\
Tautbg/3433 & $B$ & 25 & 51.4 & 1972.35 \\
Tautbg/3436 & $V$ & 25 & 51.4 & 1972.35 \\
UKST/J5193 & $B_{\mathrm{J}}$ & 75 & 67.2 & 1979.54 \\
Tautbg/5821 & $V$ & 20 & 51.4 & 1982.36 \\
Tautbg/8348 & $B$ & 32 & 51.4 & 1993.37 \\
Tautbg/8353 & $B$ & 29 & 51.4 & 1993.38 \\
\hline
\end{tabular}

\section{Observations and measurements}

The plates used for the proper motion determination (see Scholz et al. 1996) were taken with the Tautenburg, Palomar and UK Schmidt telescopes and are listed in Table 1. All plates were measured with the APM measuring machine in Cambridge/UK (Kibblewhite et al. 1984). For more details of the measuring process we refer the reader to our earlier globular cluster papers (Scholz et al. 1993, 1994). A comparison of the quality of the different Schmidt plates, concerning their astrometric use is given in Scholz et al. (1996). Whereas on the Palomar and UKST plates M 5 was close to the plate edge the Tautenburg plates were taken centered on the cluster.

All measured objects were classified into stars, nonstellar objects, noise images and merged objects using the standard APM software. In the case of M 3 (Scholz \& Kharchenko 1994) we included only the objects classified on all plates as stellar in the membership determination. Here we are interested in a more complete sample of cluster members, i.e. including the large number of objects in the cluster region misclassified as non-stellar (galaxies or merged objects) due to crowding effects. Of course we have to consider the much lower accuracy in the proper motions and in the two-colour photometry of these objects. 

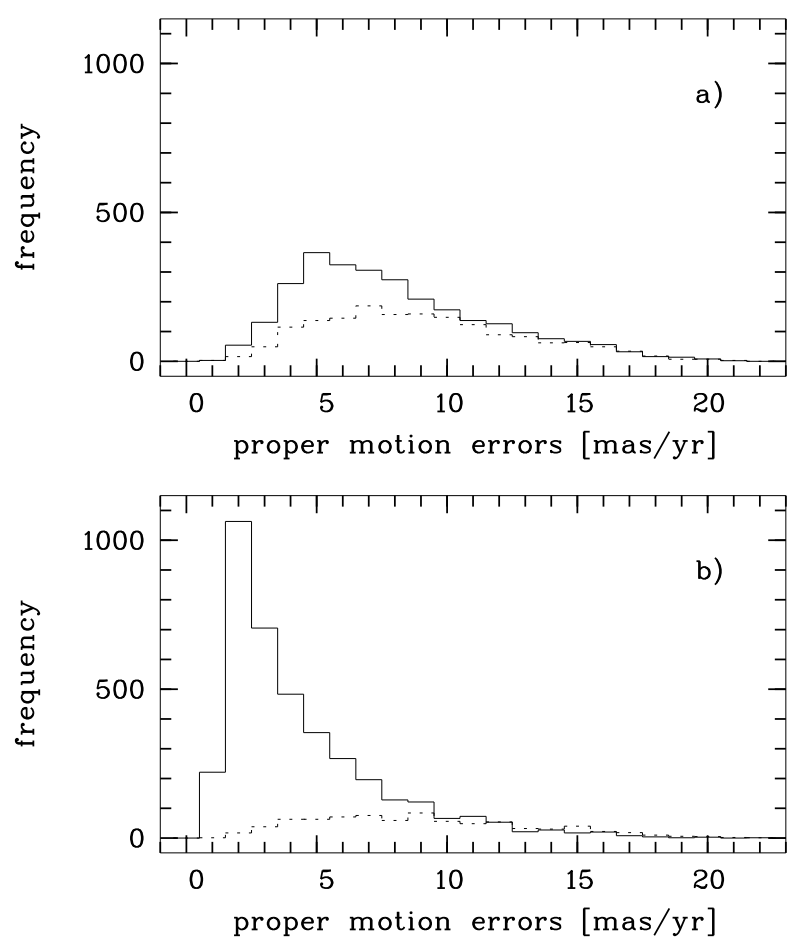

Fig. 1. Distribution of the proper motion errors for images with stellar (solid line) and non-stellar (dotted line) classification a) all objects excluded in the membership determination, i.e. all faint objects $(B>19)$ and all objects within 5 arcmin from the cluster centre $\mathbf{b})$ the objects used in the determination of membership probabilities (in the solution of (3)) i.e. all objects with 5 arcmin $<r<50$ arcmin and with $B<19$. Note the difference between the proper motion errors $\varepsilon_{\mu}$ shown in this diagram and the proper motion dispersions $\sigma_{\mu}$ obtained in the membership analysis

\section{Determination of proper motions and $B, V$ magnitudes}

As the plate covering the maximum overlap field of all plates around the cluster and at the same time being one of the deepest, plate No. 5821 was selected as reference plate in the plate matching and astrometric reduction. The proper motion determination had already been described in Scholz et al. (1996). The absolute proper motions were obtained by a plate overlap solution using about 1200 background galaxies as reference objects. Before that an extensive error removal technique was applied in order to exclude systematic positional plate-to-plate distortions of the different Schmidt plates and the known errors of the measuring machine (cf. Evans 1988; and Evans \& Irwin 1995).

A vector-point diagram of the absolute proper motions of all stars in the cluster region had already been included in the previous paper (Fig. 4a in Scholz et al. 1996). The histogram of the total proper motion errors in
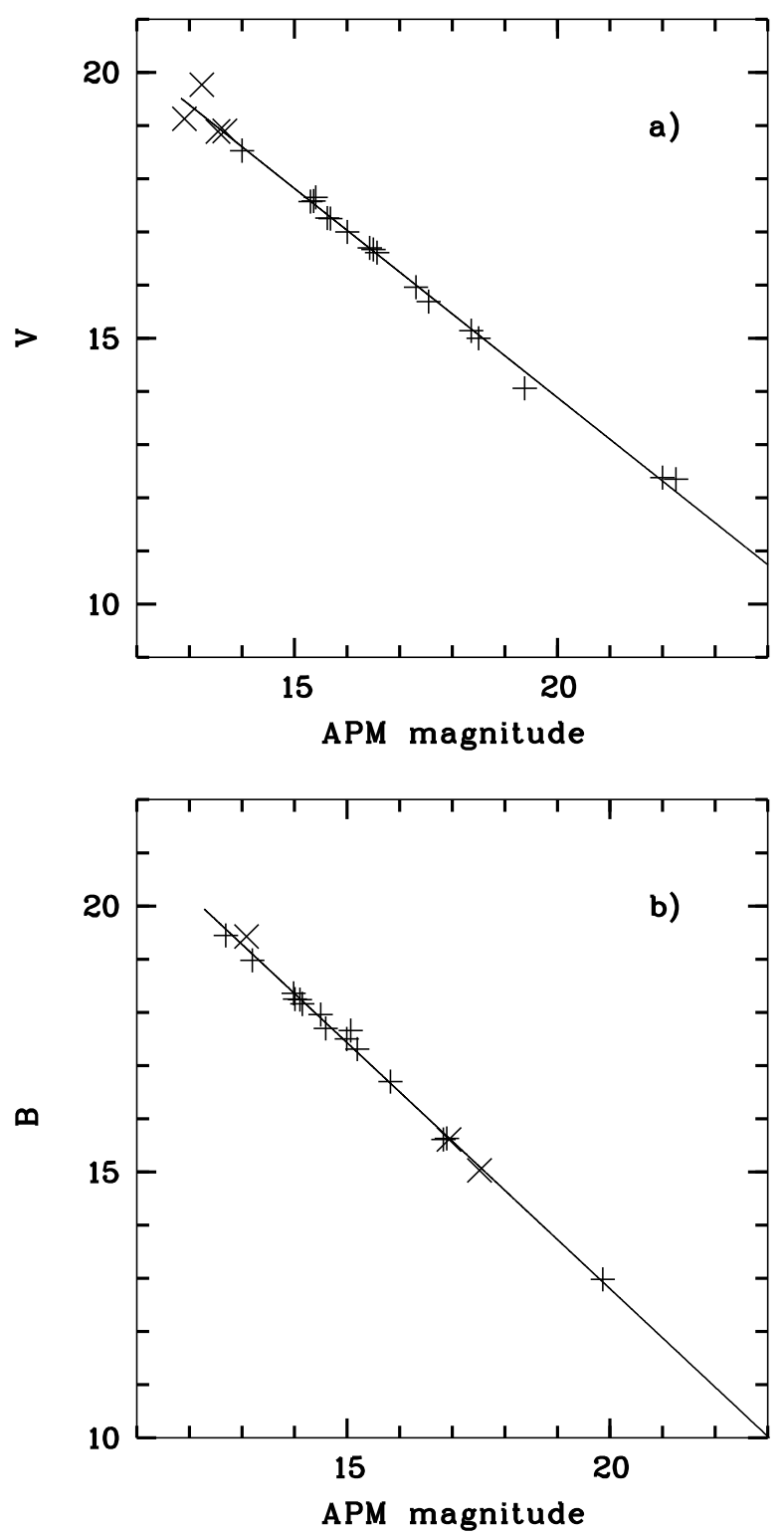

Fig. 2. Calibration of the APM magnitudes a) on the $V$ plate 5821 and b) on the $B$ plate 8348 with photoelectric standards from Arp (1962). A linear polynomial fit was used. Objects with stellar classification are plotted as + , those with non-stellar classification (not used in the fitting) are plotted as $\times$

dependence on the object classification is shown in Fig. 1. Except for the faintest stars and for the globular cluster stars in the central part of the cluster strongly affected by image crowding typical proper motion errors (peak in Fig. 1b) of 2 mas/yr were obtained. The median of the distribution of proper motion errors for stellar images shown in Fig. $1 \mathrm{~b}$ is at about $4 \mathrm{mas} / \mathrm{yr}$.

The deepest Tautenburg plates in $B$ (8348) and $V$ (5821) have been used here for a two-colour photographic 
photometry in order to compare the CMD with the astrometric membership determination. The APM magnitudes are already internally calibrated (see Bunclark \& Irwin 1983) so that they are almost linearly related to the standard photometric system. For the external calibration we used the photoelectric standards from Arp (1962) well covering the magnitude interval $11<V<19.5$ of our study. Among the 21 identified stars from Arp (1962) there were several objects classified as non-stellar on our plates due to crowding effects. The accuracy of our photographic two-colour photometry can be roughly estimated as \pm 0.1 getting worse at the faint end.

\section{Determination of cluster membership}

Cluster membership can be estimated from kinematical data (i.e. proper motions and/or radial velocities), from the number density of the stars around an assumed cluster centre, from the CMD or from a common analysis of these data which allows to obtain more reliable results. Astrometric membership probabilities from proper motion data can be computed using different criteria. After shifting the origin of the proper motion diagram to the center of the proper motion distribution of the cluster stars, Ebbinghausen (1942) applied a probability factor $P=a \cdot \varepsilon_{\mu}$ where $\varepsilon_{\mu}$ is the rms error of an individual stellar proper motion and $a$ is $\sqrt{2}, 2$ or $2 \sqrt{2}$ for, as he called, high probable, probable and low probable members, respectively. This approach can be successfully used if the proper motion distribution of the cluster members shows a concentration to a point different from the distribution of the field stars. Vasilevskis (1962) fitted the proper motion distribution with two bivariate Gaussians and calculated the membership probability for each star as the relation between the distribution functions of cluster members and of all stars. Sanders (1971) suggested to use the maximum likelyhood method for the fitting and computed distribution parameters of cluster and field stars and cluster membership probabilities. Nevertheless, the determination of membership probabilities by the method of Sanders (1971) can also lead to uncorrect results if the distribution functions of cluster and field stars are overlapping. Frequently, the proper motions of cluster and field stars separated by this method are not normally distributed. This effect is clearly visible with large numbers of stars included in the investigation (cf. the results for the globular cluster M 3 by Scholz \& Kharchenko 1994).

Especially if we use deep Schmidt plates with moderate scale and relatively small epoch differences of a few decades a membership determination based only on proper motions is difficult for distant clusters. That is due to the low significance of the individual proper motions of the cluster stars and to a large number of faint field stars which have proper motions of the same order as the cluster stars. Kharchenko \& Schilbach (1995) met these difficulties when they were trying to obtain membership probabil- ities of four open clusters located in the Saggitarius Carina spiral arm of the Galaxy. As far as the fit of two bivariate Gaussians to the proper motion distribution did not yield stable results they suggested to use the information on both proper motion and position distributions of the stars near the clusters. The parameters of cluster and field star distributions were computed in a four-dimensional space of proper motions $\left(\mu_{x}, \mu_{y}\right)$ and plate coordinates $(X, Y)$. We apply this method here to the globular cluster M 5 and compare the results with those obtained from the membership determination by using only the proper motions or only the positions of the stars.

The distribution function $F\left(\mu_{x}, \mu_{y}, X, Y\right)$ of the stars in the vicinity of the cluster can be described as the sum of two distribution functions, respectively of the field stars and of the cluster stars:

$$
F\left(\mu_{x}, \mu_{y}, X, Y\right)=F_{\mathrm{f}}\left(\mu_{x}, \mu_{y}, X, Y\right)+F_{\mathrm{c}}\left(\mu_{x}, \mu_{y}, X, Y\right) .
$$

The cluster itself can in principle also be divided into two parts - the core and the korona - with different velocity dispersions. If we speak about a core region in our membership analysis we do not mean the real cluster core in the astrophysical sense. The real core radius $r_{\mathrm{c}}$ of the cluster is less than 1 arcmin (see Table 5). This very central region of the cluster is not resolved at all on the Schmidt plates. In all membership solutions we used only the images outside a cluster radius of 5 arcmin (but computed membership probabilities for all objects). As we will see later there is no indication of two different cluster populations from our membership determination. Note that in the star counts using the whole measuring data of the Schmidt plates (see Sect. 8.1.) we applied a crowding correction in order to reach the inner regions of the cluster. The stellar population in the field is also not homogeneous as far as we observe field stars at rather different distances and, therefore, with different kinematical properties. To a first approximation we can consider two groups of stars main sequence stars with spectral classes A, F, G, K and red giants of the disk, respectively with absolute magnitudes $M_{V}=4 \ldots 6$ and $M_{V}=2 \ldots 3$. The mean distances of these two groups of stars $\bar{r}$ in the given magnitude interval differ by a factor of 5 , whereas their mean space velocities $\bar{v}$ usually differ only by a factor of 2 (Kharchenko 1980). The proper motions are proportional to $v / r$, therefore the distribution function of the field stars is again the sum of at least two components. We can neglect other field stars, i.e. high luminosity stars and stars of other luminosity classes due to their small numbers. So we get

$$
F=F_{\mathrm{f}}+F_{\mathrm{c}}=F_{\mathrm{f}}^{\text {near } *}+F_{\mathrm{f}}^{\text {distant } *}+F_{\mathrm{c}}^{\text {core }}+F_{\mathrm{c}}^{\text {korona }}
$$

The parameters of the distribution function can be determined by the maximum likelyhood method. These parameters are independent of each other in such a system of coordinates $(P, Q)$ and of proper motions $\left(\mu_{P}, \mu_{Q}\right)$, where the corresponding correlation coefficients are equal to zero. 
That means, our four-dimensional coordinate system must be rotated by angles $\Phi_{\mu}$ and $\Phi_{\text {pos }}$. These angles are determined by the condition that the correlation coefficients between the parameters reach a minimum. Assuming normal distributions of the proper motions of all stellar groups and of the coordinates of the cluster stars and a uniform distribution of the coordinates of the field stars within a radius $R_{\mathrm{f}}$ we can write the following equations in the new coordinate system for the cluster stars (core or corona):

$F_{\mathrm{c}}\left(\mu_{P}, \mu_{Q}, P, Q\right)=\frac{n_{\mathrm{c}} \cdot \mathrm{e}^{-\frac{H_{1}+H_{2}}{2}}}{4 \pi^{2}\left(\sigma_{\mu_{P_{\mathrm{c}}}} \sigma_{\mu_{Q_{\mathrm{c}}}} \sigma_{P_{\mathrm{c}}} \sigma_{Q_{\mathrm{c}}}\right)^{2}}$

and for the field stars (nearby or distant stars):

$F_{\mathrm{f}}\left(\mu_{P}, \mu_{Q}, P, Q\right)=\frac{n_{\mathrm{f}} \cdot \mathrm{e}^{-\frac{H_{3}}{2}}}{2 \pi^{2}\left(\sigma_{\mu_{P_{\mathrm{f}}}} \sigma_{\mu_{Q_{\mathrm{f}}}}\right)^{2} R_{\mathrm{f}}^{2}}$

with

$H_{1}=\left(\frac{\mu_{P}-\overline{\mu_{P_{\mathrm{c}}}}}{\sigma_{\mu_{P_{\mathrm{c}}}}}\right)^{2}+\left(\frac{\mu_{Q}-\overline{\mu_{Q_{\mathrm{c}}}}}{\sigma_{\mu_{Q_{\mathrm{c}}}}}\right)^{2}$

$H_{2}=\left(\frac{P-\overline{P_{\mathrm{c}}}}{\sigma_{P_{\mathrm{c}}}}\right)^{2}+\left(\frac{Q-\overline{Q_{\mathrm{c}}}}{\sigma_{Q_{\mathrm{c}}}}\right)^{2}$

and

$H_{3}=\left(\frac{\mu_{P}-\overline{\mu_{P_{\mathrm{f}}}}}{\sigma_{\mu_{P_{\mathrm{f}}}}}\right)^{2}+\left(\frac{\mu_{Q}-\overline{\mu_{Q_{\mathrm{f}}}}}{\sigma_{\mu_{Q_{\mathrm{f}}}}}\right)^{2}$.

If we consider the proper motions and coordinates of $N$ stars as an $N$-dimensional random vector with independent components its probability density will be determined by the likelihood function

$L\left(\boldsymbol{\mu}_{P}, \boldsymbol{\mu}_{Q}, \boldsymbol{P}, \boldsymbol{Q}, \boldsymbol{S}\right)=\prod_{i=1}^{N} F_{i}\left(\mu_{P}, \mu_{Q}, P, Q\right)$,

where

$\boldsymbol{\mu}_{P} \equiv\left\{\mu_{P_{i}}\right\}, \boldsymbol{\mu}_{Q} \equiv\left\{\mu_{Q_{i}}\right\}, \boldsymbol{P} \equiv\left\{P_{i}\right\}, \boldsymbol{Q} \equiv\left\{Q_{i}\right\}$

and $\boldsymbol{S}$ is the vector of the parameters to be determined. The function (2) reachs the maximum at $\boldsymbol{S}=\boldsymbol{S}_{\mathrm{o}}$. With these conditions we get a system of $j$ equations for the determination of the components of the vector $\boldsymbol{S}$ :

$\left.\frac{\partial \sum_{i=1}^{N} \ln F_{i}\left(\mu_{P}, \mu_{Q}, P, Q\right)}{\partial S_{j}}\right|_{S_{j 0}}=0$.

If $F_{i}$ corresponds to (1), we have $j=1,2, \ldots, 28$. After rotating the coordinate system back to the system $(X, Y)$ and $\left(\mu_{x}, \mu_{y}\right)$ the membership probabilities of cluster stars were computed as

$P_{\mathrm{c}}=\mathrm{e}^{\left(-0.5 \frac{H_{4}+H_{5}}{4}\right)}$ with

$H_{4}=\frac{\left(\mu_{x}-\bar{\mu}_{x_{\mathrm{c}}}\right)^{2}}{\sigma_{\mu_{x_{\mathrm{c}}}}^{2}}+\frac{\left(\mu_{y}-\bar{\mu}_{y_{\mathrm{c}}}\right)^{2}}{\sigma_{\mu_{y_{\mathrm{c}}}}^{2}}$

and

$H_{5}=\frac{\left(X-\bar{X}_{\mathrm{c}}\right)^{2}}{\sigma_{X_{\mathrm{c}}}^{2}}+\frac{\left(Y-\bar{Y}_{\mathrm{c}}\right)^{2}}{\sigma_{Y_{\mathrm{c}}}^{2}}$.

Membership probabilities $P_{\mathrm{c} \mu}$ and $P_{\mathrm{cp}}$, respectively computed only from proper motions or only from the positions are

$P_{\mathrm{c} \mu}=\mathrm{e}^{-0.5 \cdot \frac{H_{4}}{2}}$,

$P_{\text {cp }}=\mathrm{e}^{-0.5 \cdot \frac{H_{5}}{2}}$.

Table 2. Parameters of distribution functions

\begin{tabular}{cccc}
\hline parameter & M 5 & near field stars & distant field stars \\
\hline$n$ & 1573 & 2224 & 1276 \\
$\bar{X}[\operatorname{arcmin}]$ & -3.63 & & \\
$\sigma_{X}$ & 8.94 & & \\
$\bar{Y}[\operatorname{arcmin}]$ & +1.84 & & \\
$\sigma_{Y}$ & 8.93 & & \\
$\overline{\mu_{x}}[\mathrm{mas} / \mathrm{yr}]$ & +5.51 & -0.41 & -2.52 \\
$\sigma_{\mu_{x}}$ & 5.39 & 13.60 & 5.96 \\
$\overline{\mu_{y}}[\mathrm{mas} / \mathrm{yr}]$ & -7.75 & -5.80 & -1.72 \\
$\sigma_{\mu_{y}}$ & 5.02 & 12.63 & 5.59 \\
\hline
\end{tabular}

Stars with cluster membership probabilities $P$ higher than 61,14 and 1 per cent are located at distances less than $1 \sigma, 2 \sigma$ and $3 \sigma$ from the maximum of the fourdimensional distribution function. Respectively, they were considered as high probable, probable and low probable cluster members.

The number of unknown quantities in the system of Eqs. (3) is too large for getting a stable solution even with large numbers of stars. Additional assumptions concerning for instance the symmetric distribution of the cluster members in the space of coordinates and proper motions (cf. Kharchenko \& Schilbach 1995) are needed. In our case we did not made assumptions concerning the symmetry of the proper motion dispersion or of the dispersion in the positional distribution of the cluster stars, but we could reduce the number of the components of the distribution function (1). As expected we did not find any indication of two different distributions of cluster stars in the region investigated $(r>5$ arcmin) in preliminary membership determinations using only the proper motions. With a heliocentric distance of $7.6 \mathrm{kpc}$ (Peterson 1993) for M 5 and the probably small internal velocities of the cluster 

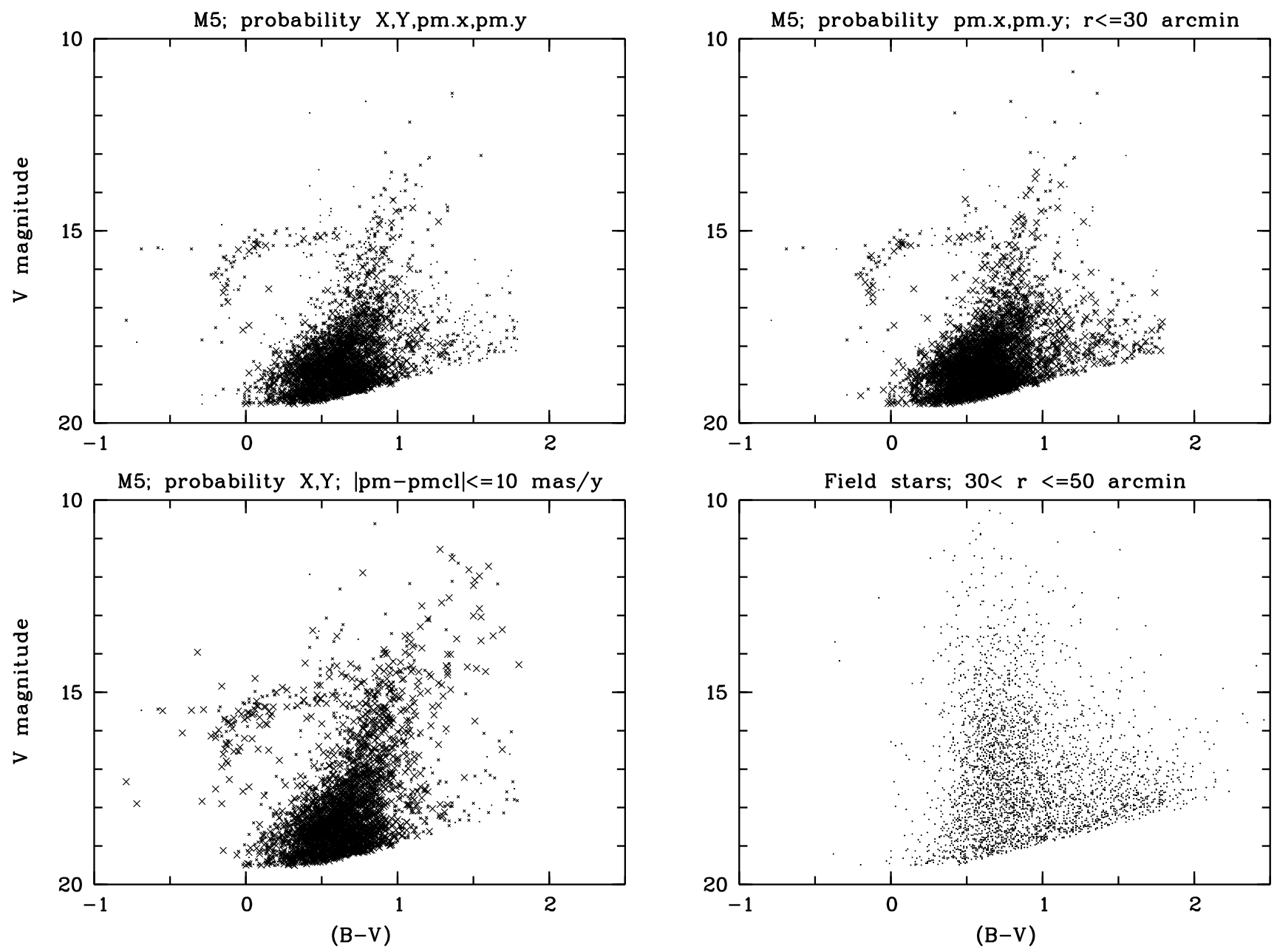

Fig. 3. Colour-magnitude diagrams for cluster members obtained in the four-dimensional space of proper motions and coordinates (upper left), in the two-dimensional membership determinations using only the proper motions (upper right) or using only the coordinates (lower left) and for the field stars (lower right). The high probable cluster members, probable members and low probable members (respectively, within $1 \sigma, 2 \sigma$ and $3 \sigma$ from the centre of the distribution) are marked by crosses, small crosses and dots, respectively. In the lower right diagram the dots represent all stars in an annulus around the cluster

stars $(\sim 2 \mathrm{~km} / \mathrm{s})$ corresponding to about $0.06 \mathrm{mas} / \mathrm{yr}$ we must observe similar proper motion distribution functions of a cluster core and korona dominated by the rms errors of the stellar proper motions. On the other side we did also not find different parameters for two cluster populations in the membership solution using only the coordinates. Therefore, we excluded the core distribution function $F_{\mathrm{c}}^{\text {core }}$ from Eq. (1) and left with 19 unknowns in the case of a membership solution by use of proper motions and coordinates (i.e. for the cluster stars: the mean proper motion components and their dispersions, the centre coordinates and their dispersions and the number of cluster stars; for near field stars: their mean proper motion components and dispersions and their number; for distant field stars: their mean proper motion components and dispersions and their number).
For the solution of Eq. (3) we excluded obvious nonmembers, i.e. stars located outside a cluster radius of 50 arcmin and high proper motions stars with total proper motions larger than 40 mas/yr. We also excluded stars with large colour indices $(B-V)>1.8$. In order to get more reliable results we did not use the faintest stars with $B>19$ and the stars inside a cluster radius of 5 arcmin, strongly affected by image crowding on the Schmidt plates. By the last two conditions we excluded most of the stars with large proper motion errors. Among the remaining 5073 stars we determined 1573 cluster stars, 1276 distant field stars and 2224 near field stars with the parameters given in Table 2. 


\section{Comparison of membership probabilities with colour-magnitude diagram}

Figure 4 shows the CMDs for the cluster stars determined in the four-dimensional space of proper motions and coordinates with membership probabilities according to Eq. (4) in comparison to the two-dimensional membership determinations using only the proper motions (Eq. 5) and only the coordinates (Eq. 6). For the two-dimensional membership determination we applied additional restrictions concerning the coordinates or proper motions of the stars. The fourth CMD represents the field stars located far outside the cluster region.

Table 3. Cluster parameters dependent on membership probabilities

\begin{tabular}{ccccc}
\hline $\begin{array}{c}\text { membership } \\
\text { probability }\end{array}$ & $N$ & $\begin{array}{c}r_{\max } \\
{[\operatorname{arcmin}]}\end{array}$ & $\begin{array}{c}\overline{\mu_{x}} \\
{[\mathrm{mas} / \mathrm{yr}]}\end{array}$ & $\begin{array}{c}\overline{\mu_{y}} \\
{[\mathrm{mas} / \mathrm{yr}]}\end{array}$ \\
\hline$P_{\mathrm{c}}>61 \%$ & 218 & 9.5 & $+5.57 \pm 0.20$ & $-7.85 \pm 0.20$ \\
$P_{\mathrm{c}}>14 \%$ & 1025 & 19.3 & $+5.81 \pm 0.19$ & $-7.92 \pm 0.17$ \\
$P_{\mathrm{c}}>1 \%$ & 1562 & 27.6 & $+4.73 \pm 0.19$ & $-7.29 \pm 0.17$ \\
\hline
\end{tabular}

Comparing the CMDs we see that the membership determination in the four-dimensional space of proper motions and coordinates provides the best results. The "best" CMD was selected visually on the basis of the smallest spread of the cluster members along the known lines of globular cluster CMDs. In comparison to the CMD of Arp (1962) who's photoelectric magnitudes were taken as reference in our photographic photometry we obtain cluster members in three "non-characteristic" regions:

1) $(B-V)>1.1$ and $V>16.5$,

2) $(B-V)<0.2$ and $V>18.0$,

3) $(B-V)<0.0$ and $V>16.0$.

We checked these stars for their concentration towards the cluster centre. All three groups of stars show a strong concentration so that we conclude they are cluster members.

\section{Mean absolute proper motion and cluster radius from membership analysis}

The mean absolute proper motion of the cluster given in Scholz et al. (1996) had been determined without the knowledge of membership probabilities for individual stars. The contamination with field stars in the cluster region was considered statistically on the basis of the number density and mean proper motion of the field stars outside the cluster. The ratio of the number of cluster stars to the number of field stars in the cluster region defined in Scholz et al. (1996) as $4 \operatorname{arcmin}<r<14$ arcmin was $845 / 215$. Note that in this case only the faint objects with stellar classification were used in the averaging of the proper motions. Stars brighter than $V=17.5$ were not used in order to correspond to the magnitude interval of the reference galaxies and to prevent possible magnitude dependent systematic errors.

Here we look according to the conditions used in the membership calculations only for objects with $r>5$ arcmin and $B<19$. As a compromise between the number of stars used in the averaging and the expected contamination with field stars the mean cluster proper motion given in the second row of Table 3 seems to represent the most reliable result. Comparing the vector-point proper motion diagrams of the stars listed in Table 3 we find the most symmetric distribution in the case of $P_{\mathrm{c}}>14 \%$. In all three cases the median values of $\mu_{x}$ tend to be slightly larger than the mean values given in Table 3 . For $P_{\mathrm{c}}>$ $14 \%$ the median of $\mu_{x}$ is at $6.15 \mathrm{mas} / \mathrm{yr}$. Nevertheless, there remains a somewhat smaller value of $\overline{\mu_{x}}$ in comparison to the result $\left(\overline{\mu_{x}}, \overline{\mu_{y}}\right)=(+6.7 \pm 0.5,-7.8 \pm 0.4)$ of Scholz et al. (1996). This difference may be the result of a small magnitude dependent systematic error in $\mu_{x}$, previously not detected. In the other proper motion component, $\mu_{y}$ a magnitude equation had already been mentioned and corrected for in Scholz et al. (1996). A smaller value of $\overline{\mu_{x}}$ (e.g. +5.8 instead of +6.7 mas/yr) does not change the character of the Galactic orbit of M 5 , unless it leads to a decrease in the total value of the space motion of the cluster (from 421 to $391 \mathrm{~km} / \mathrm{s}$ ), and therefore, to a smaller apogalactic distance (44 instead of $61 \mathrm{kpc}$ ) of the cluster (Odenkirchen 1996). Nevertheless, the former result of Scholz et al. (1996) remains more reliable, as far as it dit not include the bright cluster stars probably affected by a systematic magnitude dependent error with respect to the mean magnitude of the reference galaxies $(\bar{V}=19)$.

As result from the membership analysis in the fourdimensional space of coordinates and proper motions we find cluster members at a maximum radius of about 28 arcmin (cf. Tables 3 and 4). If we plot the CMDs for all cluster members within $3 \sigma$ from the centre of the fourdimensional distribution function $\left(P_{\mathrm{c}}>1 \%\right)$ in three different zones around the cluster (Fig. 4), so we obtain in all three zones a CMD which is different from the CMD of the field stars (cf. Fig. 4). With increasing distance from the cluster centre the contamination with field stars increases. However, even in the outer zone $(19.3 \operatorname{arcmin}<r<27.6$ arcmin) the diagram looks typical of a globular cluster CMD.

Table 4 shows the mean proper motions of cluster members determined in the four-dimensional membership analysis (with $P_{\mathrm{c}}>1 \%$ ) and of cluster members obtained from a membership analysis using the proper motions only (with $P_{\mathrm{c} \mu}>14 \%$ ) in comparison to the non-members with total proper motions less than 18 mas/yr. In the inner zone between 5 and 10 arcmin there is a higher number density of non-members. This effect is due to the larger 
Table 4. Cluster and field stars parameters in radial zones around M 5

\begin{tabular}{|c|c|c|c|c|c|c|c|c|c|}
\hline \multirow{2}{*}{$\begin{array}{c}\text { Zone } \\
\Delta r \\
{[\operatorname{arcmin}]}\end{array}$} & \multicolumn{3}{|c|}{$P_{\mathrm{c}}>1 \%(\mathrm{M} 5)$} & \multicolumn{3}{|c|}{$P_{\mathrm{c} \mu}>14 \%(\mathrm{M} 5)$} & \multicolumn{3}{|c|}{$P_{\mathrm{c}}=0$ (field stars) } \\
\hline & $N$ & $\begin{array}{c}\overline{\mu_{x}} \\
{[\mathrm{mas} / \mathrm{yr}]}\end{array}$ & $\begin{array}{c}\overline{\mu_{y}} \\
{[\mathrm{mas} / \mathrm{yr}]}\end{array}$ & $N$ & $\begin{array}{c}\overline{\mu_{x}} \\
{[\mathrm{mas} / \mathrm{yr}]}\end{array}$ & $\begin{array}{c}\overline{\mu_{y}} \\
{[\mathrm{mas} / \mathrm{yr}]}\end{array}$ & $N$ & $\begin{array}{c}\overline{\mu_{x}} \\
{[\mathrm{mas} / \mathrm{yr}]}\end{array}$ & $\begin{array}{c}\overline{\mu_{y}} \\
{[\mathrm{mas} / \mathrm{yr}]}\end{array}$ \\
\hline $5-10$ & $\overline{792}$ & $+5.71 \pm 0.28$ & $-7.82 \pm 0.27$ & 573 & $+5.88 \pm 0.23$ & $-7.86 \pm 0.25$ & 47 & $-2.74 \pm 1.46$ & $+1.58 \pm 1.33$ \\
\hline $10-12.5$ & 207 & $+5.80 \pm 0.47$ & $-7.69 \pm 0.38$ & 166 & $+6.28 \pm 0.47$ & $-7.91 \pm 0.42$ & 11 & $-8.04 \pm 2.57$ & $-0.01 \pm 2.95$ \\
\hline $12.5-15$ & 140 & $+4.11 \pm 0.62$ & $-6.82 \pm 0.62$ & 98 & $+5.20 \pm 0.61$ & $-7.67 \pm 0.58$ & 15 & $-3.96 \pm 1.58$ & $-0.43 \pm 1.71$ \\
\hline $15-17.5$ & 118 & $+3.47 \pm 0.69$ & $-6.24 \pm 0.52$ & 78 & $+4.14 \pm 0.68$ & $-7.20 \pm 0.65$ & 21 & $-4.72 \pm 1.54$ & $+0.28 \pm 1.52$ \\
\hline $17.5-20$ & 104 & $+2.88 \pm 0.65$ & $-6.38 \pm 0.58$ & 77 & $+4.29 \pm 0.67$ & $-6.91 \pm 0.69$ & 29 & $-5.07 \pm 1.16$ & $+1.64 \pm 1.16$ \\
\hline $20-22.5$ & 91 & $+1.56 \pm 0.61$ & $-6.09 \pm 0.62$ & 72 & $+3.05 \pm 0.72$ & $-6.97 \pm 0.68$ & 35 & $-4.37 \pm$ & $+1.59 \pm 1.42$ \\
\hline $22.5-25$ & 74 & $+1.78 \pm 0.67$ & $-6.39 \pm 0.57$ & 66 & $+2.48 \pm 0.74$ & $-6.47 \pm 0.70$ & 50 & $-4.38 \pm 1.03$ & $-1.54 \pm 0.96$ \\
\hline $25-27.6$ & 36 & $+2.86 \pm 0.62$ & $-6.28 \pm 0.62$ & 69 & $+3.01 \pm 0.73$ & $-5.43 \pm 0.72$ & 65 & $-3.90 \pm 0.79$ & $-0.90 \pm 0.86$ \\
\hline
\end{tabular}

individual proper motion errors in the inner cluster region resulting from stronger crowding effects. With increasing cluster

radius we can see a systematic change in the mean proper motion of the cluster members in direction of the mean proper motion of the field stars (cf. Table 2). But comparing the data of Tables 4 and 2 even in the outer zone between 25 and 27.6 arcmin we see a significant difference between the mean cluster and mean field star proper motion for both membership probabilities $P_{\mathrm{c}}$ and $P_{\mathrm{c} \mu}$.

In Table 4 we compared the results for $P_{\mathrm{c}}>1 \%$ and $P_{\mathrm{c} \mu}>14 \%$ in order to investigate the most reliable membership probabilities, particularly in the outer cluster region. With $P_{\mathrm{c}}>14 \%$ there are no cluster members outside 20 arcmin from the cluster centre. On the other side with $P_{\mathrm{c} \mu}>1 \%$ the mean proper motion components change more rapidly from +5.73 to $+0.20 \mathrm{mas} / \mathrm{yr}$ for $\overline{\mu_{x}}$ and from -8.07 to $-4.79 \mathrm{mas} / \mathrm{yr}$ for $\overline{\mu_{y}}$, respectively from the $5-10$ arcmin zone to the $25-27.6$ arcmin zone.

The number of field stars $n_{\mathrm{f}}$ contaminating the mean cluster motion can be estimated from

$\mu_{\mathrm{fc}}=\frac{n_{\mathrm{c}} \mu_{\mathrm{c}}+n_{\mathrm{f}} \mu_{\mathrm{f}}}{n_{\mathrm{c}}+n_{\mathrm{f}}}$

where $\mu_{\mathrm{fc}}$ is the mean proper motion of the cluster contaminated with field stars. The number of field stars included in the numbers of cluster members given in Cols. 2 and 5 of Table 4 is about 20 per cent and does not exceed 30 per cent even in the outer zone $(19.3$ arcmin $<r<27.6$ arcmin). Therefore, we conclude from the membership determination alone that the cluster radius is not less than 27.6 arcmin.

\section{External comparison of M 5 membership probabilities}

The membership analysis of Rees (1993) includes 515 stars in the magnitude interval $10.9<V<15.7$ covering the central $12 \times 12 \operatorname{arcmin}^{2}$ region of M 5 . Only 33 per cent of these stars are located outside of 5 arcmin from the cluster centre. Allowing half an arcsec differences in the coordinates we matched 149 stars from the catalogue of Rees (1993) with our sample.

The relative proper motions of Rees (1993) are extremely accurate: with total proper motion errors ranging from 0.15 mas/yr in the inner cluster region $(r<8$ arcmin) to about $0.30 \mathrm{mas} / \mathrm{yr}$ for the stars in the outer cluster region of his catalogue $(r \sim 12$ arcmin $)$. He achieved a further improvement in comparison to the former work of Cudworth (1979) by including more plates and by a modified proper motion and membership derivation. The proper motion accuracy of our catalogue is more than 10 times worse (cf. Fig. 1) with a different trend (better accuracy in outer cluster region compared to the inner region) caused by image crowding. Due to the much better proper motion accuracies of Rees (1993) we can use his membership probabilities as $100 \%$ true reference.

In order to compare our membership probabilities with those of Rees (1993) we did not exclude the inner cluster region $(r<5$ arcmin) but instead decided not to use the stars with large total proper motion errors. From 104 stars with total proper motion errors of less than 7 mas/yr there were 82 members and 22 non-members according to Rees (1993). Figure 5 shows the histogram of our membership probabilities for these true members (solid lines) and nonmembers (dashed lines). In addition to the membership probabilities $P_{\mathrm{c}}, P_{\mathrm{c} \mu}$ and $P_{\mathrm{cp}}$ we plotted the histogram of the averaged membership probabilities $\left(P_{\mathrm{c} \mu}+P_{\mathrm{cp}}\right) / 2$.

If we define from our membership analysis all stars above a certain membership probability level as members and all stars below that level as non-members, so we obtain in the best case (at the level $P_{\mathrm{c}}=2 \%$ ) 85 per cent of all 104 stars having the same membership classification as given by Rees (1993). The next best segregation is obtained with $\left(P_{\mathrm{c} \mu}+P_{\mathrm{cp}}\right) / 2=22 \%$ (correct membership classification for 82.5 per cent of the stars). As far as only a region close to the cluster centre could be used for an external comparison of our membership probabilities the reliability of our membership probabilities in the outer cluster region can only be estimated on the basis of the 

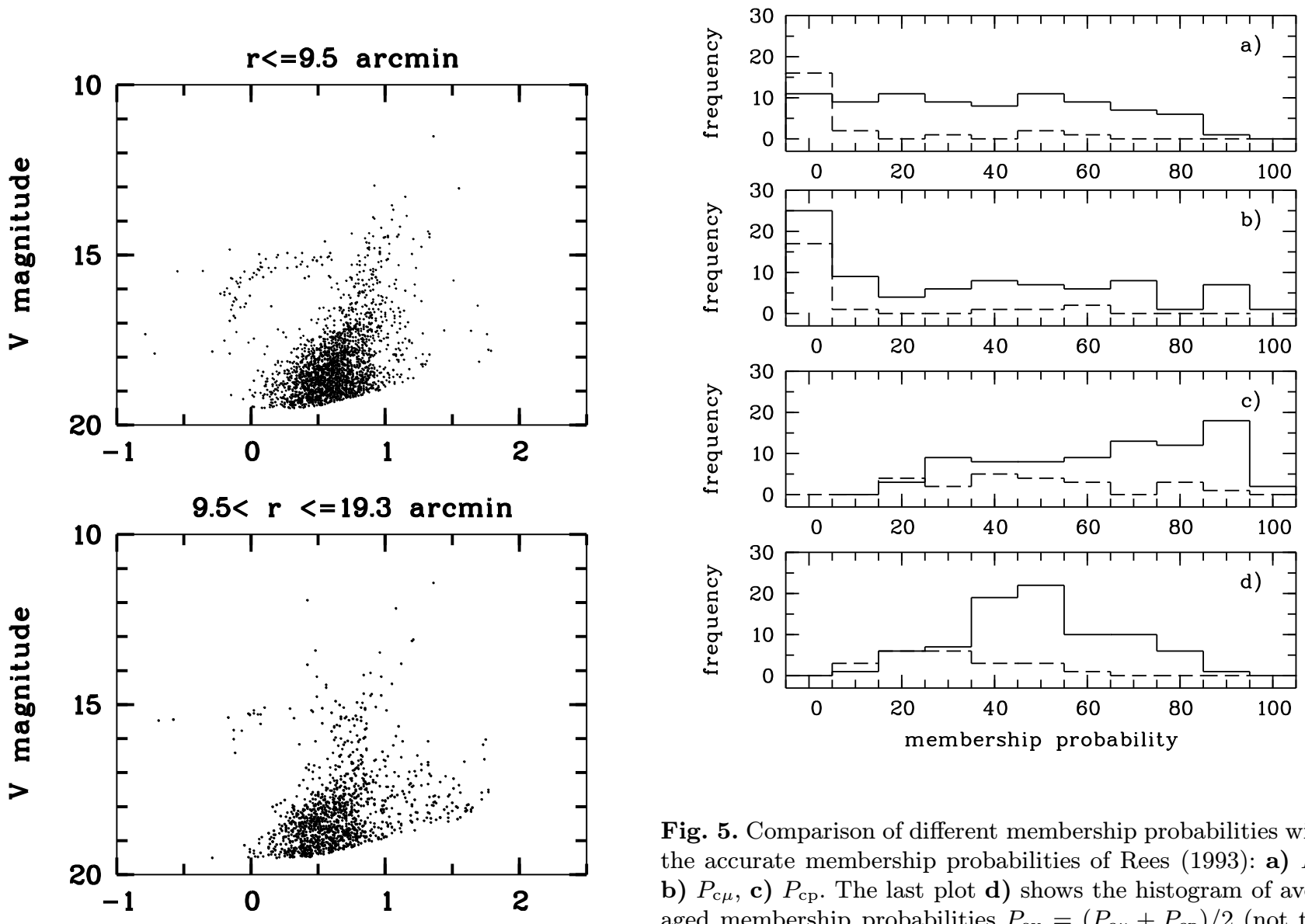

Fig. 5. Comparison of different membership probabilities with the accurate membership probabilities of Rees (1993): a) $P_{\mathrm{c}}$, b) $P_{\mathrm{c} \mu}$, c) $P_{\mathrm{cp}}$. The last plot $\left.\mathbf{d}\right)$ shows the histogram of averaged membership probabilities $P_{\mathrm{av}}=\left(P_{\mathrm{c} \mu}+P_{\mathrm{cp}}\right) / 2$ (not the average of the histograms b) and $\mathbf{c})$ ). The solid line shows the histogram of our membership probabilities for cluster members from Rees (1993) whereas the dashed line shows the histogram of our membership probabilities for non-members from Rees (1993). From 149 cross-identifications with the catalogue of Rees (1993) only the objects with total proper motion errors of less than 7 mas/yr are included in the comparison. As far as only a region close to the cluster centre could be used for the comparison, our membership probabilities based only on the proper motions (affected by larger errors due to crowding effects near the cluster centre) show the worst result

knowledge of the mean proper motion and number density of the field stars using relationship (7) and by comparison with the CMD of the cluster (see previous section).

\section{Star counts and profile fitting}

\subsection{Counts of complete measurements on different Schmidt plates}

In order to increase the accuracy of the tidal radius of M 5

Fig. 4. Colour-magnitude diagram for cluster members with $P_{\mathrm{c}}>1 \%$ in three radial zones: $r<9.5$ arcmin, $9.5<r<19.3$ and $19.3<r<27.6$ (cf. with CMD of field stars = lower right CMD in Fig. 3)

known from Peterson \& King (1975), Bahcall \& Hausman (1976), Kron et al. (1984) and Trager et al. (1995) we used the full APM scans of 8 Schmidt plates (cf. Table 1) for star counts (Lehmann 1996). The automated counts are characterised by a higher statistical significance in the 


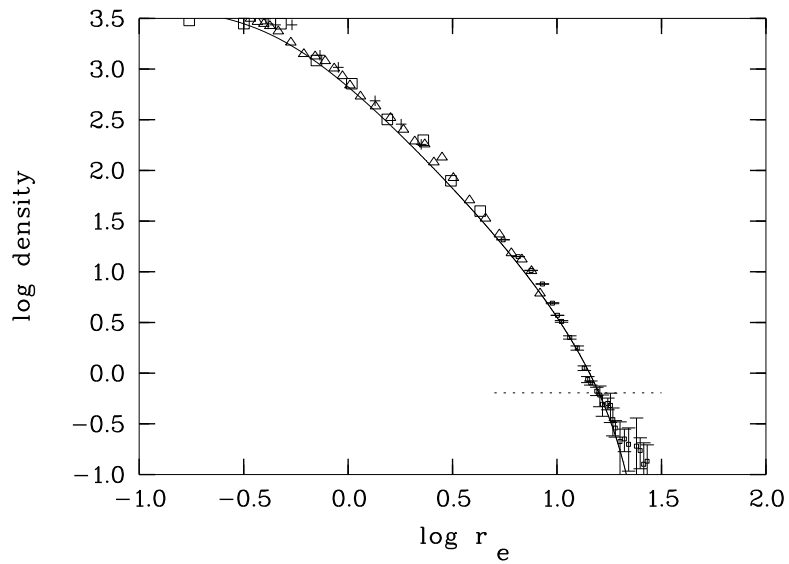

Fig. 6. Projected surface density profile of M 5. Logarithm of surface density in stars per $\operatorname{arcmin}^{2}$ versus logarithm of the mean annulus radius $r_{\mathrm{e}}$ [arcmin]. The line shows the best fit with the empirical density law from King (1962). In the fitting procedure only the star counts (dots with error bars) and the data from King (1966) (crosses) were used. Also shown are the data from Kron et al. (1984) (triangles) and from Kron \& Mayall (1960) (boxes). The dotted line indicates the background surface density

outer region of globular clusters in comparison with the human detection. Due to crowding effects the central cluster region could not be resolved in a range of $1-3$ arcmin (in dependence on the plate scale, seeing and limiting magnitude). There were also significant crowding effects in the range of $3.0<r_{\mathrm{e}}<14.0 \operatorname{arcmin}\left(r_{\mathrm{e}}\right.$ - the mean radius of the annulus). Therefore we applied a luminosity dependent crowding correction based on the luminosity function in the outer cluster region and the statistical probability of overlapping star images (for more details see Lehmann 1996).

Note that the crowding correction was not applied in the APM on-line reduction (improved image detection in crowded fields of Irwin 1985) as done in Grillmair et al. (1995). Instead here we used standard APM measurements, originally carried out for astrometry. The crowding correction converted the high number of images with nonstellar classification appearing in the inner annuli around the cluster centre on the basis of their pixel area into additional numbers of stellar images. For this purpose the luminosity function of stellar images in the outer cluster region (not affected by crowding) obtained after the subtraction of the background was assumed to be valid in the inner cluster region, too. The subtracted background surface density was determined from a large region adjacent to the cluster in an annulus between 25 and 50 arcmin from the cluster centre.

The projected surface density profiles of M 5 from the different plates were matched to a single profile and cor- related with photoelectric and electronographic surface brightness measurements from Kron \& Mayall (1960), King (1966) and Kron et al. (1984). Because of the spread of the surface brightness data in the innermost part of the cluster we used the above mentioned data in different combinations for the fit of the whole profile by the empirical density law of King (1962). The best fit was obtained by combining our star counts with the data of King (1966). By fitting with the Levenberg-Marquardtmethod (Press et al. 1992) we got the tidal radius of M 5 $r_{\mathrm{t}}=27.93 \pm 0.80$ arcmin, the core radius $r_{\mathrm{c}}=0.44 \pm 0.04$ arcmin and the concentration parameter $c=1.80 \pm 0.04$. Whereas the core radius agrees well with the data from Trager et al. (1995) the tidal radius from our profil is 1.7 arcmin lower than their value (see Table 5).

Table 5. Structural parameters $r_{\mathrm{t}}, r_{\mathrm{c}}$ and $c$ of $\mathrm{M} 5$ as obtained by different authors and methods

\begin{tabular}{cccl}
\hline $\begin{array}{c}r_{\mathrm{t}} \\
{[\operatorname{arcmin}]}\end{array}$ & $\begin{array}{c}r_{\mathrm{c}} \\
{[\operatorname{arcmin}]}\end{array}$ & $c$ & reference \\
\hline 28.84 & 0.48 & 1.78 & Peterson \& King $(1975)^{1)}$ \\
16.03 & 0.32 & 1.70 & Bahcall \& Hausman $(1976)^{1)}$ \\
24.74 & 0.44 & 1.75 & Kron et al. $(1984)^{2)}$ \\
29.64 & 0.40 & 1.87 & Trager et al. $(1995)^{3)}$ \\
27.93 & 0.44 & 1.80 & Lehmann $(1996)$, this paper $^{4)}$ \\
\hline
\end{tabular}

${ }^{1}$ star counts by eye

2 electronographical photometry

3 CCD photometry

4 automated star counts.

Figure 6 shows for $r_{\mathrm{e}}>r_{\mathrm{t}}$ an increased surface density which may be an indication for a tidal tail - a halo of unbounded stars around the globular cluster as recently discussed by Grillmair et al. (1995). These authors investigated 12 Galactic globular clusters, mainly at southern declinations, measured on UK Schmidt plates by means of the APM and found extra-tidal wings in the density profiles for the majority of clusters in their sample. The seven globular clusters studied by Lehmann (1996) on the basis of Tautenburg, Palomar and UK Schmidt plates also scanned with the APM are located at northern declinations. There is only one cluster (M 15) included in both studies. It is interesting that Lehmann (1996) also found indications of tidal tails for 5 clusters.

Grillmair et al. (1995) investigated the nearby cluster background in more detail in order to decide whether the increased density at $r>r_{\mathrm{t}}$ is physically existent or an artifact of an incorrect background subtraction. Here we check the reliability of the tidal tail of M 5 obtained in the counts of all objects measured on 8 different Schmidt plates using the member counts for the King profile fitting. Tucholke (1992) proposed this method for globular 
clusters with rich or variable background (and/or foreground) of field stars.

\subsection{Member counts with different membership probabilities}

The member counts which we present below do not go as deep as the counts of the full scans of the plates combined into one profile. This is due to the restriction of the sample of objects used in the proper motion study and in the two-colour photometry, i.e. only the objects measured on the reference plate (5821) and on a second plate (8348) were investigated. No further restrictions concerning the magnitude range of the stars used in the member counts were made.

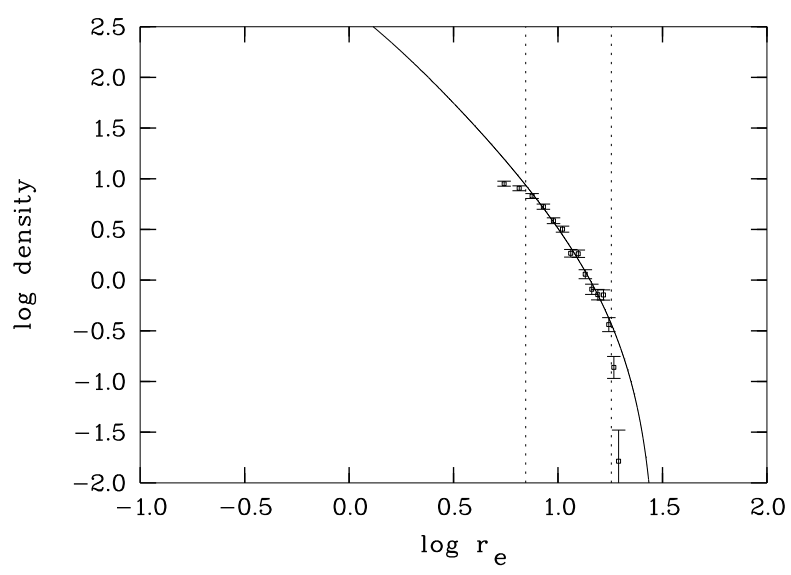

Fig. 7. Density profile of M 5 from counts of cluster members with membership probabilities $P_{\mathrm{c}}>14 \%$ (membership determination using coordinates and proper motions). Logarithm of surface density in stars per $\operatorname{arcmin}^{2}$ versus logarithm of the mean annulus radius $r_{\mathrm{e}}$ [arcmin]. The line shows the best fit with the empirical density law from King (1962). The dotted lines show the radial fitting range

For the member counts we first used the stars with membership probabilities $P_{\mathrm{c}}>1 \%$ and $P_{\mathrm{c}}>14 \%$ obtained in the four-dimensional analysis including the coordinates. These membership probabilities are the most reliable ones, although the assumption of a normal distribution of the coordinates of the cluster stars (see Sect. 4) pre-determines the shape of the density profile. For $P_{\mathrm{c}}>14 \%$ the fit with the empirical density law of King (1962) was more accurate. The results are shown in Fig. 7 and in Table 6.

For the member counts based on membership probabilities obtained only from the proper motions we present the result for the tidal radius for both using all stars with $P_{\mathrm{c} \mu}>14 \%$ and with $P_{\mathrm{c} \mu}>1 \%$ in Table 6 . As one can see the radial fitting range is larger than in the case of the

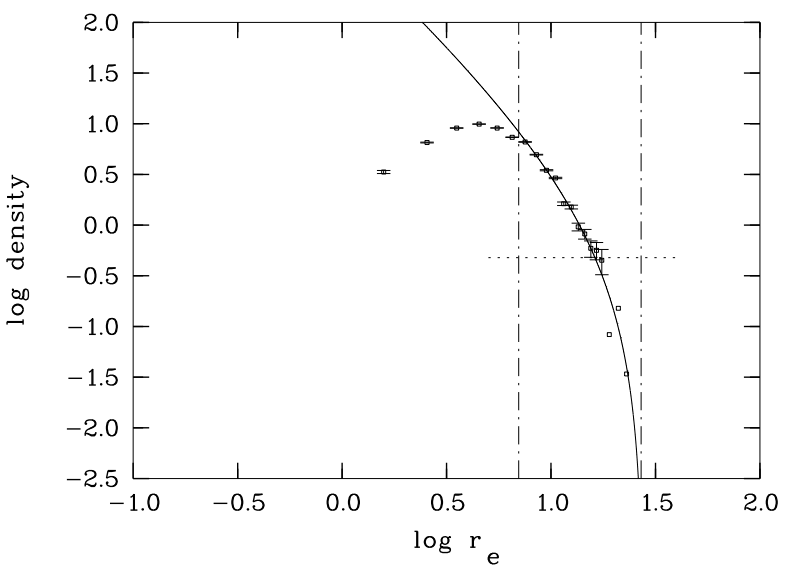

Fig. 8. Density profile of M 5 from counts of cluster members with membership probabilities $P_{\mathrm{c} \mu}>1 \%$ (membership determination using proper motions only). Logarithm of surface density in stars per $\operatorname{arcmin}^{2}$ versus logarithm of the mean annulus radius $r_{\mathrm{e}}$ [arcmin]. The line shows the best fit with the empirical density law from King (1962). The dotted line shows the subtracted background of field stars with $P_{\mathrm{c} \mu}>1 \%$ outside of 35 arcmin, the dotted-dashed lines show the radial fitting range

Table 6. M 5 tidal radius from member counts

\begin{tabular}{cccc}
\hline $\begin{array}{c}\text { membership } \\
\text { probability }\end{array}$ & $\begin{array}{c}r_{\mathrm{t}} \\
{[\operatorname{arcmin}]}\end{array}$ & $\begin{array}{c}\text { radial fitting } \\
\text { range [arcmin] }\end{array}$ & $\chi_{\text {red }}^{2}$ \\
\hline$P_{\mathrm{c}}>14 \%(2 \sigma)$ & $30.31 \pm 3.15$ & $7.0-18.0$ & 2.40 \\
$P_{\mathrm{c} \mu}>14 \%(2 \sigma)$ & $27.13 \pm 2.21$ & $7.0-27.0$ & 0.86 \\
$P_{\mathrm{c} \mu}>1 \%(3 \sigma)$ & $27.68 \pm 2.12$ & $7.0-28.0$ & 0.65 \\
\hline
\end{tabular}

$P_{\mathrm{c}}-$ membership from proper motions and positions $P_{\mathrm{c} \mu}$ - membership from proper motions only.

member counts using the four-dimensional membership analysis. Whereas in the member counts with $P_{\mathrm{c}}>14 \%$ there was no background to be subtracted, here we had to consider the number of field stars with proper motions similar to those of the cluster stars.

The values of $r_{\mathrm{t}}$ obtained in the member counts are well in agreement with those of Peterson \& King (1975), Trager et al. (1995) and of the complete scans of all Schmidt plates used in this project combined to one profile (Lehmann 1996). But there is no indication of a tidal tail for M 5 as found from the complete scans of the plates (Lehmann 1996). This discrepancy may be due to the different magnitude limits of the full counts and of the member counts. 


\section{Conclusion}

We have extended the membership investigation of the globular cluster M 5 to fainter stars and to the outer cluster regions by the use of Schmidt plates completely scanned with the APM measuring machine. For our membership study we used a four-dimensional space of proper motions and coordinates and obtained mean absolute proper motions and dispersions for three distribution functions of the cluster stars, near field stars and distant field stars by the maximum likelyhood method. Unless the high accuracy of membership investigations based on proper motion studies from plates with much better astrometric scale and longer epoch differences can not be achieved, we find a correlation of our membership results with the colour-magnitude diagram of the cluster. The reliability of our membership determination is estimated between 70 and 85 per cent from both the statistical contamination with field stars and from an external comparison with the very accurate membership probabilities in the catalogue of bright cluster stars of Rees (1993).

If we compare the mean absolute proper motion of the cluster obtained from the membership analysis with that determined without the knowledge of membership probabilities of the stars (Scholz et al. 1996) we mention a somewhat lower value of $\overline{\mu_{x}}$. This difference can be explained as a small magnitude dependent systematic error. In Scholz et al. (1996) only the faint stars were used in the determination of the mean cluster motion in order to correspond to the magnitude interval of the reference galaxies and to prevent such magnitude dependent systematic errors.

From the full scans of the different Schmidt plates (5 Tautenburg, 2 Palomar, 1 UKST) with the APM measuring machine we obtained a tidal radius of M 5 $r_{\mathrm{t}}=27.93 \pm 0.80$ arcmin. Using the additional information of the membership determination we determined $r_{\mathrm{t}}=30.31 \pm 3.15$ arcmin from the member counts based on the four-dimensional membership analysis (proper motions and coordinates) and $r_{\mathrm{t}}=27.68 \pm 2.12$ arcmin from counting the cluster members determined only from their proper motions. An indication of a tidal tail as recently discussed by Grillmair et al. (1995) for 12 other globular clusters was found in the counts of the complete scans of 8 different Schmidt plates used in this project but not confirmed by the member counts. This is probably caused by the lower magnitude limit $(B \sim 20.0)$ of the membership determination in comparison to the combined counts of the complete measurements on 8 plates $(B \sim 21.5)$.

Acknowledgements. We thank the Tautenburg, Palomar and UKST Observatories for supplying the plates and Mike
Irwin for measuring them on the APM. N. Kharchenko thanks the Deutsche Forschungsgemeinschaft for financial support. We thank the referee, Dr. J. Colin for his helpful comments and suggestions.

\section{References}

Allen C., Santillan A., 1991, Rev. Mex. Astron. Astrofis. 22, 255

Arp H., 1962, ApJ 135, 311

Bahcall N.A., Hausman M.A., 1977, ApJ 213, 93

Bunclark P.S., Irwin M.J., 1983, in: Rolfe E.J. (ed.) Proc. Internat. Colloq. Statist. Meth. Astron., ESA SP-201, p. 195

Cudworth K.M., 1979, AJ 84, 1866

Cudworth K.M., Hanson R.B., 1993, AJ 105, 168

Ebbinghausen E.G., 1942, AJ 50, 1

Evans D.W., 1988, PhD Thesis, Cambridge

Evans D.W., Irwin, M., 1995, MNRAS 277, 820

Grillmair C.J., Freeman K.C., Irwin M., Quinn P.J., 1995, AJ 109, 2553

Herzog A.D., Illingworth G.D., 1977, ApJS 33, 55

Irwin M., 1985, MNRAS 214, 575

Irwin M., Trimble V., 1984, AJ 89, 83

Kharchenko, N., 1980, Astrometriya i Astrofizika 40, 44

Kharchenko N., Schilbach E., 1995, Astron. Nachr. 316, 91

Kibblewhite E., Bridgeland M., Bunclark P., Cawson M., Irwin M., 1984, in: Capaccioli M. (ed.) Proc. Conf. Astron. with Schmidt-Type Telesc. Reidel Publ., p. 89

King I.R., 1962, AJ 67, 471

King I.R., 1966, AJ 71, 276

King I.R., Hedemann E.,Jr., Hodge S.M., 1968, AJ 73, 456

Kron G.E., Hewitt A.V., Wasserman L.H., 1984, PASP 96, 198

Kron G.E., Mayall N.U., 1960, AJ 65, 581

Lehmann I., 1996, Diploma Thesis, AIP Potsdam, A\&A paper (in preparation)

Odenkirchen M., 1996 (private communication)

Peterson C.J., 1993, ASP Conf. Ser. 50, 337

Peterson C.J., King I.R., 1975, AJ 80, 427

Press H.W., Teukolski S.A., Vetterling W.T., Flannery B.P., 1992, Cambridge University Press, Numerical Recipes in FORTRAN, 2nd. Edition

Rees R.F. Jr., 1993, AJ 106, 1524

Sanders W.L., 1971, A\&A 14, 226

Scholz R.-D., Kharchenko N., 1994, Astron. Nachr. 315, 73

Scholz R.-D., Odenkirchen M., Irwin M., 1993, MNRAS 264, 579

Scholz R.-D., Odenkirchen M., Irwin M., 1994, MNRAS 266, 925

Scholz R.-D., Odenkirchen M., Hirte S., et al., 1996, MNRAS 278,251

Scholz R.-D., Schmidt K.-H., 1992, Astron. Nachr. 313, 45

Trager S.C., King I.R., Djorgovski S., 1995, AJ 109, 218

Tucholke H.-J., 1992, A\&A 264, 513

Vasilevskis J., 1962, AJ 67, 699 